In these skeletons and skulls of the dasy- nesie to be taken now and then, and somewri and thylacinus, the most carnivorous times a dose of pult. jalapi and submur. of all the indigenous mammalia of New $h y d r$, accorling to circumstances, but I Holland and Yan Diemen's Land, we per- never use mercury alone as an internal or ceire nearly the same characters in the a constitutional remedy.

teeth, in the deficiency of the orbits, in: I chiefly, however, wish to describe my the elevation, strength, and magnitude, of treatuent of the same kind of warts. the rygomatic arch, and in the general, When at Whitehaven I was once erceeddetails of the skelcton which we find in the ingly annoyed and disappointed in the order caritivora, to which we next pio- use of the $n$. argenti to them. I nau cced. Ther all present eight incisors, came one day to the dispensary with however, in the upper jow, while the car-1 warte all aromd the corona glandis, prenivorous inon-marsupial quadrupeils have senting a complete circular fringe of them. only six. Their true molares are all tuber- : le hat had gonorhea, but the discharge culated internally like the single carnivo- was nearly well. He was under the inrous tooth in the upper jaw of feline ani- flucnce of mercury when he applied, havmals. These are the pyrmy lions and ing previously been salivated by a drugtigers of the rast and isolated continent of gists. I first cut a piece from each of the Australasia.

\section{APPLICATION OH}

MERCURT, LOYOR AMNONIE ACETATIS, AXD PYROLIGNEOUS ACID,

TO

\section{VENEREAL AND GONORRHOAL ULCLRS AND WARTS.}

\section{To the Elitor of THE LAxCET.}

Sin,-Dr. Wallace s clinical lecture on cases of renereal disease, priblished in your excellent Joumal, Ebuary the 15 th, induces the to make a few oiservations on the local trcatment of velrereal and gonowhoal uleers and warts. I am not at all surprised that the nitrate of silver heals a primary renereal uker socner than the black was!, since the nercury necessarily nadergoes a partial chemis! decomposition when nixed with linewater, and thus is no:mal efeet nipon! both to the disyenary and to the workthe sore nay be greatly diminished. Set house, I mate trial of it. Sereral pait does heal those sores. When I was ofir- tients there applied to me with this kind

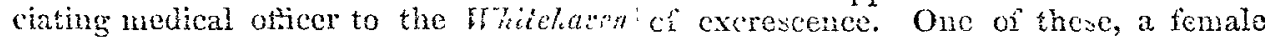
Dispens'y in 1825 , I repentedly heuled ahont seventeen or ciohtecu years of age, gonorheral and venerel vlcers by the had everal wat's and warty erorescences local application of the submit. hydr.' about the anus and interior surface of the alone, and now ber to say that I con- labia pudenda, vaying from the size of a sider this remedy to possess twa advan- kiduey-benn to a half-crown piece, actages orer the $n$ argenti; fist, its appli- companied with gonormoen. A lotion and cation is unattended by pain; anis, $z$ - sulphas magnesia were prescribed for her, condly, it absorbs any funid matter pre- with direetions to apply locally the folsent, and by this means prevents iritiation in the surrounding parts. I do nor ever rememoer secondary symptoms coning on subsequently to its application. I invariably direct the patient to wash the vencreal or gonorrhœal ulcer's three times a day with a little warm water, to dry them well, and then apply the sabmur. hydr., ordering a dose of the sulphat. mag-

\section{lowing liquilis: :-}

\section{R Acial Pyrolyguei : $\mathrm{i}$;}

Carb. Amrizonice a. s. ut ad satis saturandim.

I shall only add that the warts were removed by these means with surprising rapidity, and that she was soon free from all complaint. In my practice this liquid 
has also proved a most powerful and effcacious remedy for warts on the hands. I direct my patients, first, to pare the excrescences down with a sharp knife or razor as much as possible without causing them to bleed, and then apply the liquid. The oftener it is used the sooner the warts will be remored. They should be pared down about every second day. I am, Sir, yours very respectfully,

E. Wriktnson.

Aspatria, Cumberland, March 29, 1834.

\section{CASES OF HERNIA.}

\section{To the Editor of ThE Lancer.}

Sin,-Although the following cases have nothing very unusual in them, yet as I believe a plain statement of thern will not be without good, perhaps you will have the kindness to insert them in your journal, at a convenient time. The bad state of the omentum, and its removal with impunity, in the first case, - the universal adhesion of the intestine and its separation with safety in the second,--and the peculiar situation of the hernia and testis, together with the almost sphacelated state of the intestine in the third, are the points which make them interesting.

I am, Sir, your obedient servant, JONATHAN MONCKTON.

Brenchly, Kent, March, 1934.

Case 1.-On the 7 th of June, 1833, I saw Mrs. Cox, of Five Oaks, and found her labouring under femoral hernia. I gave her four grains of purified opium, and the same quantity of camphor, which produced (as I have ever found it to do) the most desirable state of faintness and exhaustion, with profuse perspiration. Notwithstanding the remarkable efficacy which in our practice attends this remedy, the hernia could not be returned, and 1 consequently proceeded to the operation in the usual way. I found a small knuckle of intestine, and about two ounces of omentum. The latter had lost its natural texture, was in a lump, and so much discoloured, that I remored it at once with the scalpel. Only one small vessel bled, which was secured with a thin single ligature, and the parts were returned without difficulty. The rext day she had distention of the ablomen, with extreme tenderness, and no stool. I gave her sj Olei Ricini, and the following :-

Ix Hydr. Submur. grs. xij; Pulv. Opii grs. iij. Ft. pil. xij. Capiat ij tertia quaque hora cum 6 ta parte mist. sequente.
R Mist. Sal. Com. Dss; Sulph. Magnesia jrj; Manna jij M. Ft. mist.

In a few dars all the symptoms had sulbsided, and she did perfectly well.

Case 2.-On the 11th January, 1834, I saw Mirs. Tester, of Pembury, who was labouring under hernia. After giving our $\mathrm{fa}$. vourite remedy, I used the taxis for more than an hour, when the tumour, the size of a walnut, disappeared. Ordered haustuspurgans. Nextmorning the report was, no stool or diminution of the symptoms. I went to her, and found the twmour again, which I could empty of its contents, but the intestine lay loosely without and would not return. It was evident that nothing but the operation would relieve her, I accond. ingly procured my instruments, and the ass istance of some friends, when we found a considerable knuckle of intestine down, much inflamed, and adhering to the surrounding parts so completely, that many minutes were occupied in its separation. It was then returned without trouble; violent infammation of the reluced portion of intestine followed, but gave way to leeches and the usual remedies, and she soon recovered.

Case 3.- On the 27th of January, at four in the morning, I visited John Beecher, wtat. 32 , with whom an assistant had been endeavouring, since eleven in the evening, to reduce an inguinal hernia, haviagr, in conseruence of great tenderness of the tumour, very properly bled him to $气 x r i j j$, pierious to giving the opium and canuphor; the tumour proving irreriucible. The hernia, instead of proceeding into the scrotum, had followed Poupart's liganent to near the superior anterior spinous prucess of the ilinm, and presented also a small hard tumour at its outer erge. "That, Sir," said he, "is my testicle, which las neve: been down lower than my groin." On attempting to reduce the hernia, he complained so much of soreness in the tumour and severe pain across the umbilicus that I desisted, and directed 2.5 leeches to the fumour, the warm-bath, dc., during the use of which many unsuccessful attempts were made at reduction. On proceeding to the operation again, assisterl by iny friends as before (Mr. Prine of Tunbridge Wells, and my uncle), we found about three inches of intestine down, more than half of which was of a dark olive colour. After dividing the stricture, the intestine returned with great ease; but about two ounces of thickened omentum would not return without greatcr pressure than I felt justified in using. I therefore removed it with a scalpel. Four arteries were tied, one portion of each 